\title{
TOWARDS A NOVEL AUTO-ROTATING LIDAR PLATFORM FOR CAVITY SURVEYING
}

\author{
Jordan Mitchell* \\ Offroad Robotics \\ Queen's University \\ Kingston, ON Canada
}

\author{
Joshua A. Marshall \\ Ingenuity Labs Research Institute \\ Queen's University \\ Kingston, ON Canada
}

\begin{abstract}
This paper presents the conceptual design, construction, and preliminary testing of a novel auto-rotating platform for cavity surveying. Cavity surveying involves generating a 3D model of an opening from acquired point cloud data. 3D models are used for volume estimation, stope reconciliation, dilution control, convergence monitoring, and rock mass stability analysis. In contrast to conventional cavity monitoring systems (CMS), which employ a rotating light detection and ranging (LiDAR) sensor attached to a stationary boom, the presented novel platform consists of a single point LiDAR sensor attached to an auto-rotating body as a unique solution to the cavity surveying problem. A proof of concept device consisting of a four-blade rotor and a data acquisition system was constructed and dropped from a five-metre overhang. Although auto-rotation was not reached, the set of indoor drop tests provide preliminary results that illustrate the design concept and describes the impact of design parameters.
\end{abstract}

Keywords - auto-rotation, Cavity surveying, Underground, LiDAR mapping

${ }^{*}$ Corresponding author. Email: jordan.mitchell@queensu.ca. Postal address: c/o Ingenuity Labs Research Institute, 69 Union St, Kingston, ON K7L 3N6 Canada. 


\section{Introduction}

Laser based cavity monitoring systems (CMS) have become a primary tool for monitoring underground cavities in North America [14]. These systems normally utilize a rotating light detection and ranging (LiDAR) sensor to gather 3D point cloud data about the underground cavity. The point cloud is analyzed to determine cavity volume, cavity configuration, and cavity stability. The collected data can also be used to complement softwares such as SURPAC, DATAMINE VULCAN and AutoCAD to improve cavity modelling [11]. These surveys have proven to improve mine planning and mine productivity by allowing for more accurate 3D modelling of underground cavities [13].

The original contribution of this paper rests on the concept of a novel auto-rotating platform for underground cavity surveying. This paper demonstrates preliminary test results from the deployment of a prototype platform within an indoor experimental environment.

\subsection{Cavity Monitoring Systems}

Cavity monitoring systems have many applications because they can be deployed in mine stopes, abandoned mine openings, ore passes, pillar recovery areas, backfilling areas, ore bins, tunnels, and any other cavity with limited accessibility [11]. These systems are most commonly used to conduct stope surveys in underground mines to monitor dilution. Dilution control increases mine revenues by improving the grade of blasted ore and reducing the cost involved in handling extra waste that enters the ore stream [20]. Stope surveys are conducted after a blast to assess the amount of overbreak or underbreak resulting from the blast $[10,8,15]$. Survey results allow mine operators to assess if corrective action is required to reduce dilution. Mubita [20] recorded a significant increase in stope performance by actively monitoring the stopes and iteratively correcting blasting techniques at the Konkola Mine in Zimbia.

Cavity monitoring systems have been used to gather information about potentially unsafe work environments. Liu et al. [11] used a CMS at the Sandaozhaung mine in Launchaun to identify the size, location and shape of unknown cavities that were left from previous open-stope mining techniques located under the current open pit. Liu concluded that the CMS provided an accurate 3D model of the unknown cavities and presented valuable information for guiding the disposal of the cavities, which improved the level of safety at the Sandaozhaung mine. Liu et al. [12] also used a CMS to dynamically monitor the collapse of a pillar stope at the Dongguashan Copper Mine. Multiple surveys were conducted at various locations to accurately model the irregular cavity resulting from the collapse. The information gathered from the surveys allowed mine operators to determine how to reclaim the ore remains and backfill the collapsed area.

Other uses of spatial LiDAR measurements of cavities include surface roughness 
modelling and convergence monitoring. Both Watson [26] and Fardin et al. [5] used LiDAR sensors to survey rock faces to model surface roughness. This data improved the accuracy of ventilation modelling in underground mines. Vanderbeck [25] employed a mobile LiDAR sensor to repeatedly survey mine drifts to monitor convergence. This research shows how repeated 3D surveying of cavities can be used to monitor rock deformation over time.

The traditional approach to cavity surveying is believed to have been first patented by Miller and Jacob [16] in 1993. The purpose of the invention was to provide an efficient method for measuring the dimensions of a mined stope or any other cavity. The method consists of extending a laser rangefinder at the end of a mast into an underground cavity. Once in position, the rangefinder rotates in concentric circles about the axis of the mast and collects range measurements. The series of distance measurements is then processed to synthesize a 3D point cloud of the cavity such that the shape and volume can be determined. The invention was first used by Miller et al. [17] in 1992 to measure openstope dilution. Since then, cavity monitoring systems have become a benchmark tool for monitoring underground cavities. Examples of commercially available CMSs include the MapTek I-Site 8200 laser scanner, the Renishaw Void Scanner 150 and the Optech CMS V500. At the time of writing, these systems cost approximately USD $\$ 100,000$ or more.

The main disadvantages of conventional cavity monitoring systems are the high capital costs of equipment, the stationary point of view and long survey times [12, 7]. Large rock outcrops or irregular features can block the linear scan of the LiDAR sensor causing blind spots within the 3D model. These blind spots reduce the accuracy of the 3D cavity model and hinder the ability of mine personnel to make well informed decisions. These sensors often have a range accuracy of $\pm 1-2 \mathrm{~cm}$ but the accuracy of the resulting $3 \mathrm{D}$ model is largely dependent on the environment. The $3 \mathrm{D}$ model also has a varying point cloud density as features that are farther away are represented with less points. Cavity monitoring systems collect LiDAR measurements at regular angular step changes. This results in consecutive points having a larger linear distance between them the farther they are from the sensor. Cavity surveys can take between 30 minutes to and hour including set up, data collection and disassembly. Set up includes fixing the mast to a tripod or mine truck, positioning the sensor in the middle of the cavity and calibrating the sensor. During that time the regular mine operations of the cavity being surveyed are halted. These long survey times reduce mine productivity. The aforementioned disadvantages are depicted in Figure 1.

\subsection{UAVs in the Underground}

The downsides of conventional CMSs have prompted the development of other cavity surveying tools. Several companies have employed proximity sensors mounted on unmanned aerial vehicles (UAVs) for cavity surveying. This approach involves having a trained op- 


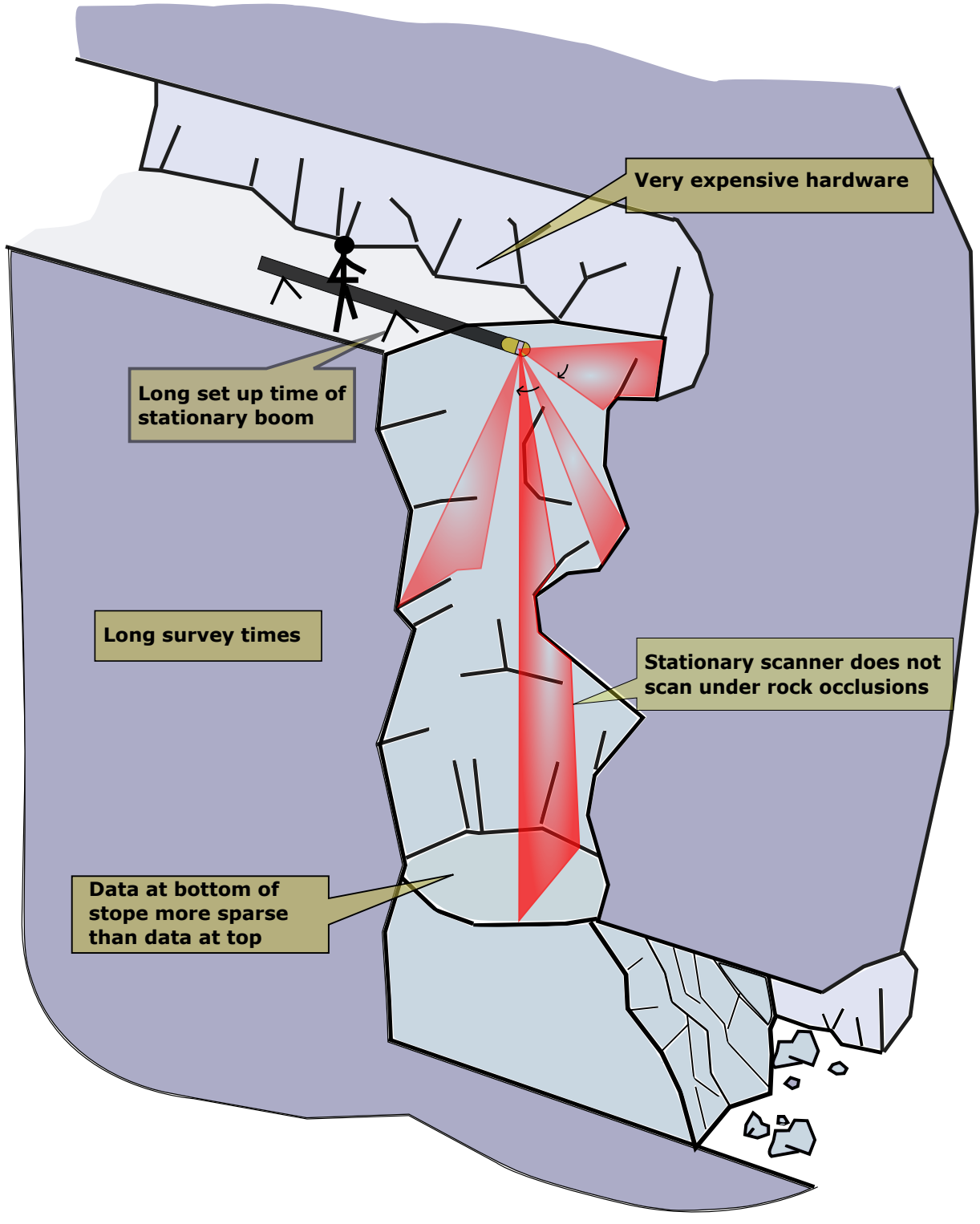

Figure 1: Infographic illustrating the limitations of using a conventional cavity monitoring system for underground surveying. 
erator navigate a UAV equipped with a range sensing payload through an underground cavity while collecting position and range data. The UAV is then returned to the operator and the data is post processed to show a map of the cavity. This method is beneficial due to the ability to scan from multiple points of view and eliminate blind spots. UAVs equipped with proximity sensing payloads offer a potentially lower cost and more accurate solution to gather 3D point clouds of underground cavities. Disadvantages of this method include the requirement for a highly trained operator to navigate the UAV and the risk of losing the UAV in an unreachable area.

There currently exists commercially available handheld mapping devices, such as the GeoSLAM ZEB REVO, that use simultaneous localization and mapping (SLAM) algorithms to map surrounding environments [21]. These systems are not suitable for large underground cavities because the cavities are not safe for human entry. Although, the same technology is currently being used to develop UAV systems capable of navigating GPS denied environments autonomously $[6,1]$. The use of UAVs underground is currently limited because most commercially available UAVs still require line of sight operation and lighting when in GPS denied environments. The confined spaces, dampness, reduced visibility, air movement and lack of control signal propagation are obstacles that hinder the operation of UAVs underground [22]. This has prompted researchers to develop unique algorithms and hardware to successfully navigate UAVs autonomously underground [24, 22, 2]. Turner [24] has conducted preliminary research towards autonomous rock face mapping with the Astec Pelican UAV and Sennersten et al. [22, 2] has conducted initial trials of beyond line of sight control with an underground UAV. At the time of writing, there is not a commercially available fully autonomous solution to cavity surveying using UAVs.

\section{Auto-rotating Platform for Cavity Surveying}

A single point LiDAR sensor attached to a rotating falling body presents a novel mechanism for gathering 3D survey data in underground cavities. A falling maple samara that auto-rotates as it falls inspired our conceptual design of an auto-rotating platform for underground cavity surveying. Auto-rotation is the state of flight where the main rotor system spins with no net power requirement [9]. Objects in auto-rotation are inherently stable with a constant descent and angular velocity [23].

By equipping an auto-rotating platform with a LiDAR payload, the platform inherently causes the sensor to rotate as it falls along the vertical extent of the cavity. Along the way, it gathers a 3D helical scan pattern of information about the surrounding environment using an on-board data acquisition system. One possible concept of operations for this design consists of an operator literally "throwing" a small, inexpensive auto-rotating device equipped with a low-cost LiDAR payload into a cavity. The device then collects 
range data from the surrounding environment, as well as information about its own motion, as it falls and rotates to the bottom. Once at the bottom of the cavity, the collected data is wirelessly transmitted back to the operators computer and the device is recovered if possible or left disposable. The data is then post processed using software to create a 3D point cloud of the cavity. The point cloud could then be uploaded into existing mine planning software programs such as Vulcan or Surpac. The concept of operations for an auto-rotating surveying device is depicted in Figure 2.

The potential benefits of using an auto-rotating device for cavity surveying in comparison with stationary surveying methods include:

1. Improved data collection;

2. Ease of operation; and

3. Lower cost of surveying.

The ability to traverse the entire vertical length of a cavity allows the device to gather range data from multiple points of view. This permits the device to survey underneath rock occlusions that would create blind spots if a stationary surveying device was used.

This design concept presents a very simple method for conducting a cavity survey. An operator is needed to turn on the device, deploy it into the cavity, and wait for it to wirelessly transmit the data after reaching the bottom. The survey time is dependent on how long it takes the device to fall to the bottom. This method is more convenient and potentially less time consuming than setting up a stationary surveying device and waiting for the device to finish a scan, although possibly less accurate. Reducing survey time improves operational efficiency because surveyors can spend more time on other operations.

It is expected that the cost of developing an auto-rotating surveying device could be significantly less than purchasing a stationary surveyor. At the time of writing, stationary CMS devices cost up to USD $\$ 100,000$ and often require monthly software fees. These high costs are the result of expensive electronics and materials. The prototype device developed during this research costed $\$ 2,400$ including materials and machining. It is estimated that replacing aluminum and carbon fibre parts with plastic and purchasing electronics in bulk could reduce this cost to less than \$1,000 CAD. This results in a significantly lower cost than conventional cavity monitoring systems.

An auto-rotating surveyor could also be lower cost and easier to deploy than using a commercially available vertical take off and land (VTOL) UAV. Commercially available UAVs require expensive on-board electronics to control and power the UAV. These electronics include: a computer, motors, a battery, lights (for underground), 2D LiDAR sensor and an inertial measurement unit (IMU). A disposable auto-rotating device does not require the same control capabilities and power requirements as a standard UAV, thus allowing less costly electronics required on-board. A potentially disposable auto-rotating 


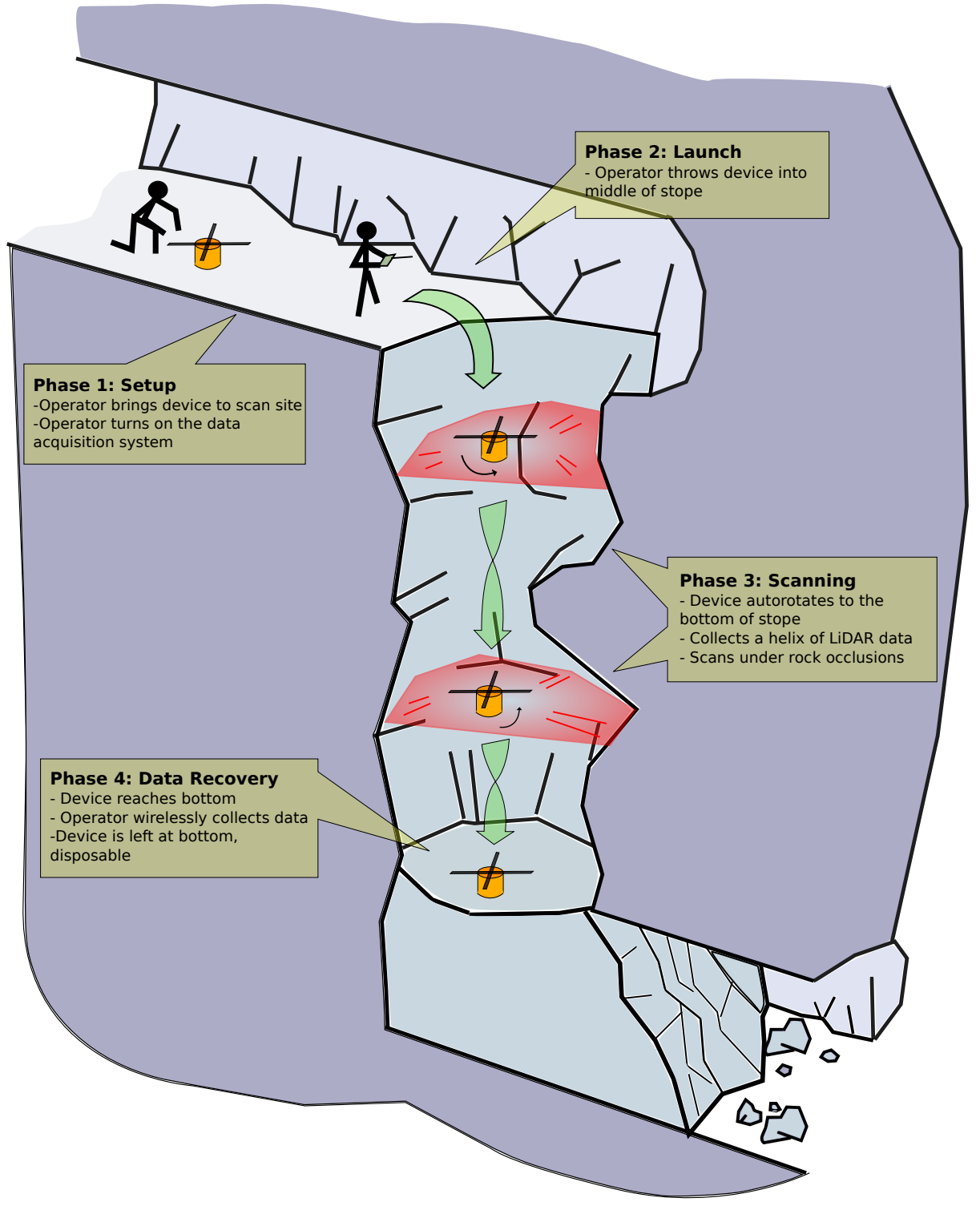

Figure 2: Operational phases include: setup, launch, scanning, and data recovery for a potentially disposable auto-rotating cavity surveying device. 
device may also be easier to deploy because once released it does not need to be controlled. An operator is not required to drive the device during its descent nor is any control algorithm required as the flight is controlled by the design of the system. These benefits of an auto-rotating design potentially make it a better tool for cavity surveying than a standard UAV.

The anticipated limitations of using an auto-rotating platform for cavity surveying include:

1. Inability to avoid obstacles;

2. Inability to be retrieved; and,

3. Inability to be deployed in certain cavities.

The proposed auto-rotating design is not controlled remotely and is intended to fall vertically through a cavity. The platform is not able to avoid obstacles on the way down. If it hits a cavity wall it is likely to disrupt the predicted flight and tumble to the bottom, thus rendering the survey useless.

The device may not be retrieved unless there is access to the bottom of the cavity. If the device is deployed in the wrong position or the data received does not adequately model the cavity, it might not be retrievable. This implies that multiple devices may be required to adequately survey a cavity.

Because this concept is such that the device falls straight down, this design is only capable of surveying cavities that have a dip angle near to ninety degrees. Otherwise, the device would clearly fall and hit the footwall and tumble along the footwall to the bottom of the cavity. The concept would also be affected by significant air velocities commonly experienced in mines due to ventilation. Significant airflow due to mine ventilation systems could cause the auto-rotating platform to deviate from its intended flight path. This is a significant limitation of the auto-rotating design, that it can only be deployed in select cavities.

\subsection{Prototype Design and Construction}

The physical design of the rotor directly relates to the flight pattern of the device. Altering the design of the airfoils in the rotor system changes the system's descent and angular velocity during flight. This, in turn, changes the helical scan pattern collected by the sensing payload. The design parameters that can be changed include:

1. UAV Mass $(m)$;

2. Rotor span-wise length $(R)$;

3. Rotor chord length $\left(c_{t i p}, c_{r o o t}\right)$; 
Table 1: Rotor design algorithm output

\begin{tabular}{rrrrrr|rrr|r}
\hline \multicolumn{7}{c}{ Design Parameters } & \multicolumn{5}{c}{ Performance } \\
\hline $\begin{array}{r}\text { Mass } \\
(\mathbf{k g})\end{array}$ & $\begin{array}{r}\text { Radius } \\
(\mathbf{m})\end{array}$ & $\begin{array}{r}\text { Tip Pitch } \\
(\mathbf{d e g})\end{array}$ & $\begin{array}{r}\text { Root Pitch } \\
\mathbf{( d e g})\end{array}$ & $\begin{array}{r}\text { Tip Chord } \\
(\mathbf{m})\end{array}$ & $\begin{array}{r}\text { Root Chord } \\
(\mathbf{m})\end{array}$ & $\begin{array}{r}\text { Descent } \\
\text { Velocity }(\mathbf{m} / \mathbf{s})\end{array}$ & $\begin{array}{r}\text { Angular } \\
\text { Velocity }(\mathbf{d e g} / \mathbf{s})\end{array}$ & $\begin{array}{r}\text { Helical Pitch } \\
(\mathbf{m})\end{array}$ & \\
\hline 0.9 & 0.4 & -3.44 & 0 & 0.04 & 0.05 & 5.4 & 9052 & 0.21 & Smallest Pitch \\
0.9 & 0.4 & -2.30 & -3.44 & 0.04 & 0.06 & 5.3 & 8823 & 0.21 & \\
0.9 & 0.4 & -3.44 & -3.44 & 0.04 & 0.05 & 5.2 & 8480 & 0.22 & \\
\hline 0.9 & 0.4 & -3.44 & 0 & 0.04 & 0.05 & 5.4 & 9052 & 0.21 & Largest Omega \\
0.9 & 0.4 & -3.44 & -2.30 & 0.05 & 0.05 & 5.5 & 8880 & 0.22 & \\
0.9 & 0.4 & -2.30 & -3.44 & 0.04 & 0.06 & 5.4 & 8880 & 0.22 & \\
\hline 0.9 & 0.5 & -1.15 & 4.58 & 0.04 & 0.05 & 4 & 4755 & 0.3 & Slowest Descent \\
0.9 & 0.5 & 1.15 & -2.30 & 0.04 & 0.05 & 4 & 4755 & 0.3 & \\
0.9 & 0.5 & 0 & 0 & 0.04 & 0.06 & 4 & 4755 & 0.3 & \\
\hline
\end{tabular}

4. Rotor pitch angle along blade $\left(\theta_{\text {tip }}, \theta_{\text {root }}\right)$; and,

5. Rotor 2D cross section shape.

These inputs must be chosen to specify the 3D helical scan pattern collected by the device.

A rudimentary algorithm was developed by Mitchell et al. [18, 19] to determine how these airfoil characteristics effect the descent and angular velocity of the device during auto-rotative descent. The algorithm was then used to select a rotor design that would allow the attached data acquisition (DAQ) system to synthesize a preliminary 3D point cloud of the surrounding cavity. Table 1 shows the output of the algorithm used to display favourable designs. The selected design is highlighted in yellow. The algorithm predicts that during equilibrium auto-rotation, this design has an angular velocity of $9052 \mathrm{deg} / \mathrm{s}$ and a descent velocity of $5.4 \mathrm{~m} / \mathrm{s}$, resulting in a helical pitch of $0.21 \mathrm{~m}$.

An on-board DAQ system was constructed to monitor the position and orientation of the device during flight as well as collect range data representing the surrounding environment. A horizontal facing LiDAR sensor was used to survey the cavity walls during descent. A vertical facing LiDAR sensor was used to monitor the vertical position and a gyroscope was selected to monitor the angular velocity during flight. The angular velocity was integrated to determine the angular position at each time stamp. An accelerometer was incorporated into the payload to monitor acceleration along the vertical axis. Table 2 shows the specifications of each of the payload instruments and Figure 3 shows the electronics used in the custom on-board DAQ system.

The rotor airfoils and payload were connected using a gimbal hub that allows for the rotor to move freely along its pitch and roll axis. This hub was inspired by the Autobody, created by Brindejonc in [4]. This gimbal hub was designed to increase the stability of the device during flight and ensure the payload hangs vertically even when the rotor system is perturbed by minor air currents.

In order to minimize weight and maintain integrity, the auto-rotating platform was constructed out of carbon fibre and aluminum. The process of constructing the device is 
Table 2: Payload instrument specifications

\begin{tabular}{|c|c|c|c|c|c|}
\hline Instrument & Vertical Lidar & Horizontal Lidar & Accelerometer & Gyroscope & Microcontroller \\
\hline Name & LiDAR-Lite V2 & $\begin{array}{l}\text { Lightware Optoelectronics } \\
\text { SF30/B }\end{array}$ & $\begin{array}{l}\text { RB-Dfr-295 } \\
\text { (ADXL345) }\end{array}$ & $\begin{array}{l}\text { Analog Devices } \\
\text { ADXRS649 }\end{array}$ & Arduino Uno \\
\hline Cost & $\$ 150$ & $\$ 640$ & $\$ 9$ & $\$ 142$ & $\$ 26$ \\
\hline Operating voltage & $5 \mathrm{~V}$ & $5 \mathrm{~V}$ & $3.3 \mathrm{~V}$ & $5 \mathrm{~V}$ & $5 \mathrm{~V}$ \\
\hline Measurement range & $40 \mathrm{~m}$ & $50 \mathrm{~m}$ & $\pm 2 \mathrm{~g}$ & $\pm 20,000 \mathrm{deg} / \mathrm{s}$ & \\
\hline Accuracy & $\pm 2.5 \mathrm{~cm}$ & $\pm 10 \mathrm{~cm}$ & & & \\
\hline Measurement frequency & $50 \mathrm{~Hz}$ & $1144 \mathrm{~Hz}$ & & & \\
\hline
\end{tabular}

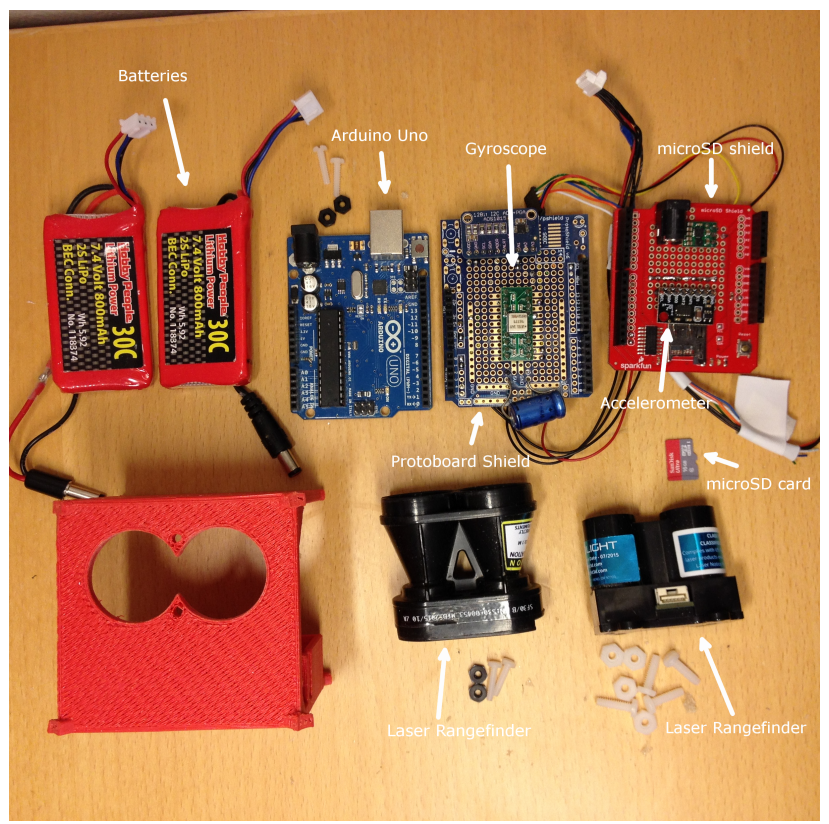

Figure 3: Electronics used to monitor the position/orientation of the device and collect cavity range measurements. 


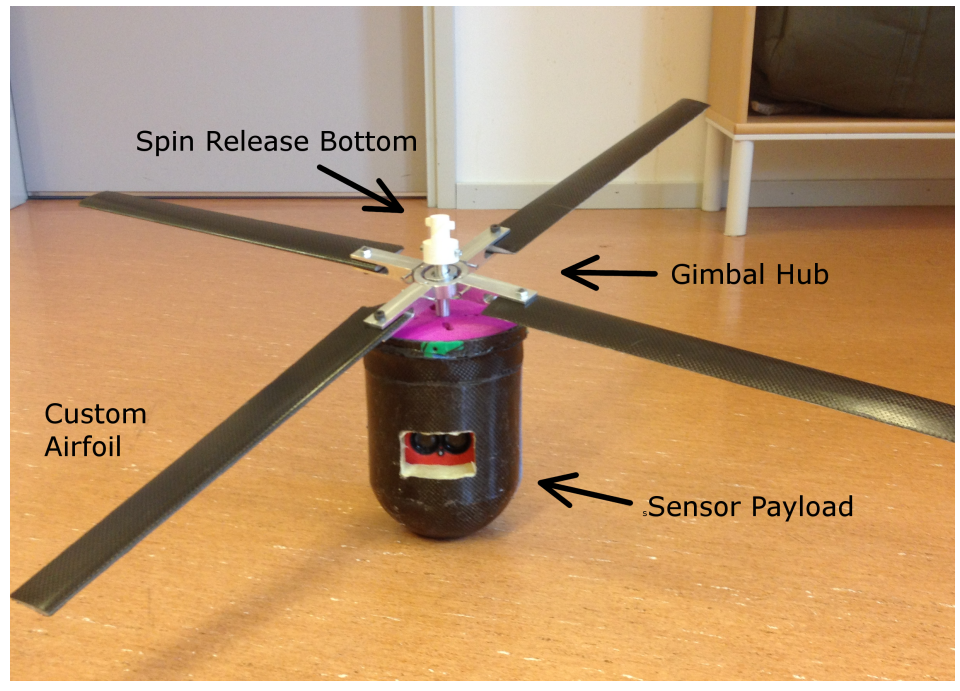

Figure 4: Initial prototype auto-rotating cavity surveyor.

discussed by Mitchell in [18]. The total cost of electronics and building materials was approximately CAD $\$ 1,700$. The fully constructed prototype is shown in Figure 4.

The constructed prototype differs from the initial concept of operations. The prototype is not deployed by an operator throwing the device. It is deployed by a spin release mechanism that angularly accelerates the device and then releases it from a boom attachment. The prototype also does not wirelessly transmit any data after use. Instead the data is collected on-board a micro SD card and retrieved after flight. These functions added additional complexity for an initial prototype and were deemed unnecessary to test the concept of an auto-rotating surveying device.

\section{Experimental Procedure}

The prototype was deployed in an laboratory environment to begin answering three basic questions:

1. Can the device maintain its integrity and withstand landing impact?

2. Does the device exhibit auto-rotation during descent?

3. Can the collected data be used to recreate a $3 \mathrm{D}$ point cloud of the environment?

The device was dropped from a height of $5 \mathrm{~m}$ over a second story stairwell overhang. An aluminium boom attached to a hand pallet truck was used to position the device in the 

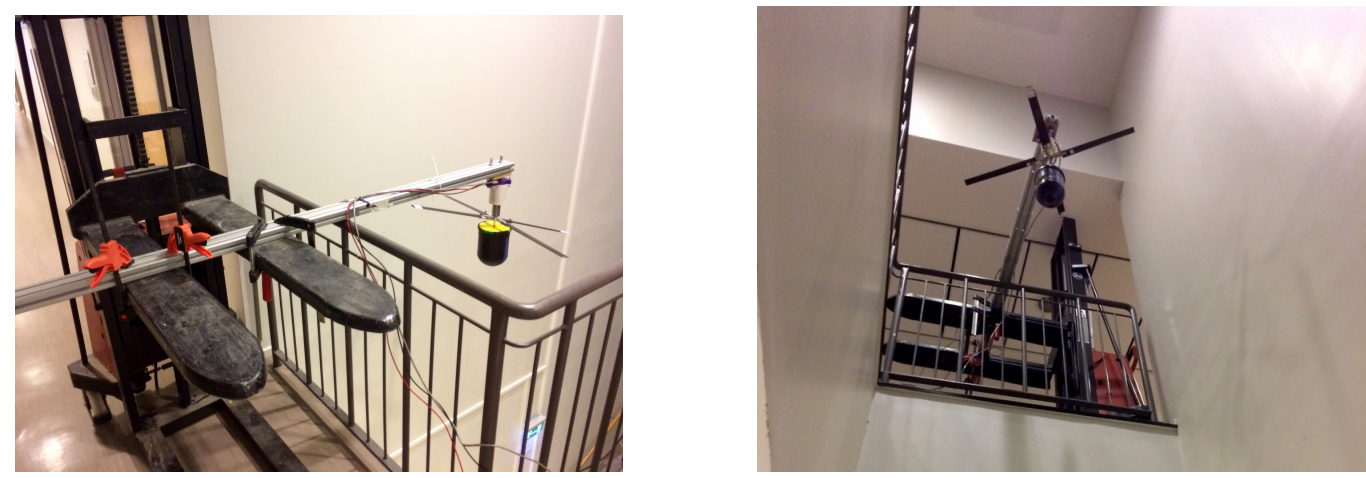

Figure 5: Top and bottom view of the experimental set up before dropping the device.

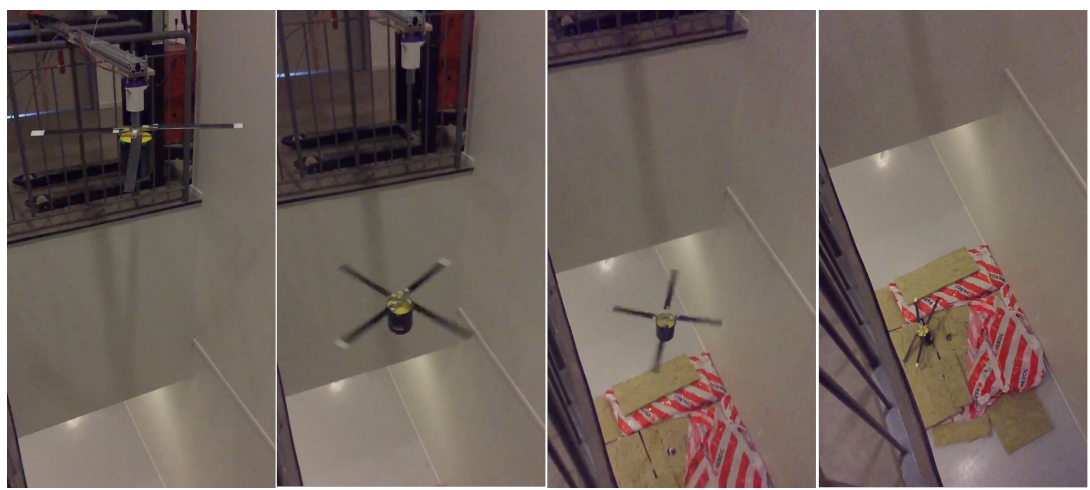

Figure 6: Falling of the auto-rotating platform.

middle of the overhang. A spin release mechanism was bolted to the end of the boom and allowed the device to be spun up and then released. Figure 5 shows the device set up prior to being released.

The prototype was dropped four times. Twice it was released at an angular velocity of roughly $2000 \mathrm{deg} / \mathrm{s}$ and twice it was released at $3300 \mathrm{deg} / \mathrm{s}$. The angular velocity upon release was varied to determine the effects of angular velocity on the 3D helical scan pattern collected. The higher angular velocity was restricted to $3300 \mathrm{deg} / \mathrm{s}$ due to the output torque of the motor used to spin the device. The device began to wobble significantly on the motor shaft and prevented higher velocities. The lower angular velocity was set at $2000 \mathrm{deg} / \mathrm{s}$ as the device began to wobble on the motor shaft at velocities greater than this. After each drop, the device was retrieved and the on-board sensor data was collected. Figure 6 displays the prototype surveying the area during its descent. 


\section{Results and Discussion}

\subsection{Device Integrity}

During each of the four drop tests the prototype was released from the spin release mechanism and fell straight down, landing on a thin foam layer to prevent marking of the floor. The device maintained its integrity for all drop tests. No parts were damaged or broken upon landing impact. The choice of materials proved to be suitable for constructing the device and the gimbal hub proved to increase the stability of the device during flight. The electronics remained intact and the collected sensor data was easily retrieved. None of the data was corrupted and permitted post processing.

\subsection{Auto-rotation}

Both the downward facing LiDAR sensor and the accelerometer showed that the device did not fall with a constant descent velocity but rather accelerated downwards as it fell. Figure 7 and Figure 8 show the collected LiDAR and gyroscope data during a trial where the device was released at $2000 \mathrm{deg} / \mathrm{s}$ and $3300 \mathrm{deg} / \mathrm{s}$, respectively. The gyroscope showed that the device decreased its angular velocity as it fell during both trials. Figure 8 shows a rapid decrease in angular velocity and vertical distance upon release. The angular velocity rapidly decreased to approximately $2600 \mathrm{deg} / \mathrm{s}$ as the motor stalls at the moment of release. The angular momentum of the device causes it to detach from the stalled motor shaft. Friction between the release mechanism and the stalled motor caused the initial decrease in angular velocity. The large negative spike in vertical distance is due to the device wobbling upon release and the downward facing lidar sensor pointing towards the wall instead of the ground. Because the device did not fall with a constant descent or angular velocity during the $5 \mathrm{~m}$ drop it did not exhibit equilibrium auto-rotation.

The device did not exhibit auto-rotation during descent, potentially due to the low height from which it was dropped and the low angular velocity at which it was released. Dropping the device from only $5 \mathrm{~m}$ did not allow the UAV enough time to reach terminal velocity. auto-rotation was observed by Brindejonc [4] when the Autobody was released from a hot air balloon, thus one might expect that the height from which our device was dropped was simply too low in comparison to its size. The device was also not released at its predicted angular velocity due to limited motor torque. If the device was rotating at its equilibrium angular velocity prior to being released it may have reached equilibrium auto-rotation during the $5 \mathrm{~m}$ descent.

The goal of achieving auto-rotation during descent soon after deployment could potentially be achieved by reducing the size and mass of the current device. By reducing the size and mass, less energy would be required from the air to induce auto-rotation. autorotation would, in this case, be achieved at a smaller descent velocity and would reduce 


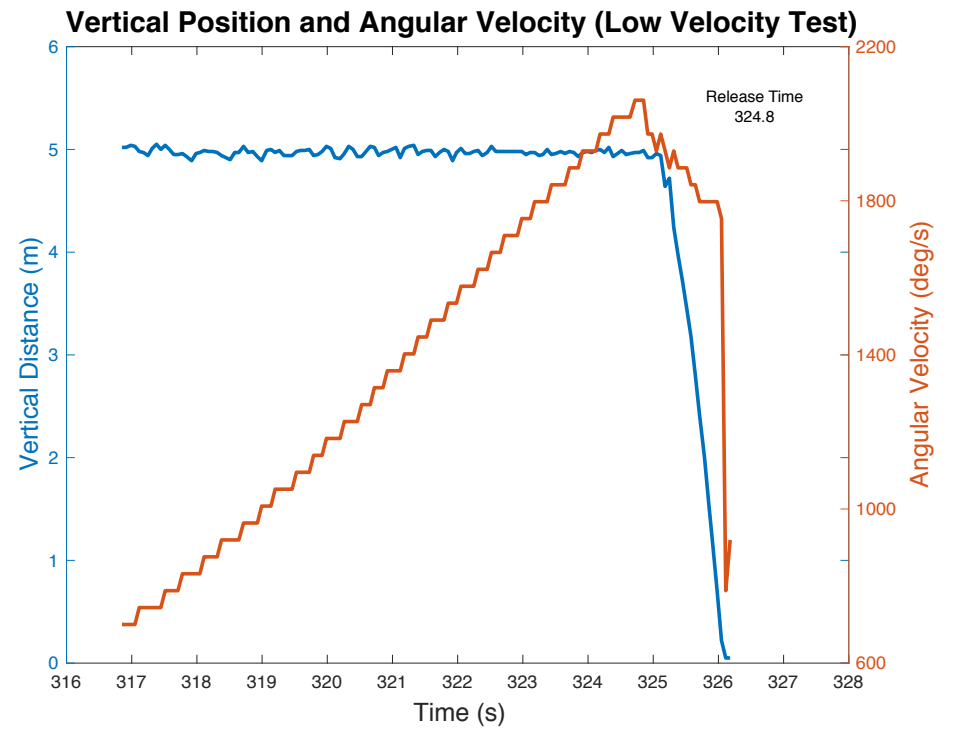

Figure 7: Raw LiDAR and angular velocity data from first drop test, released at $2000 \mathrm{deg} / \mathrm{s}$.

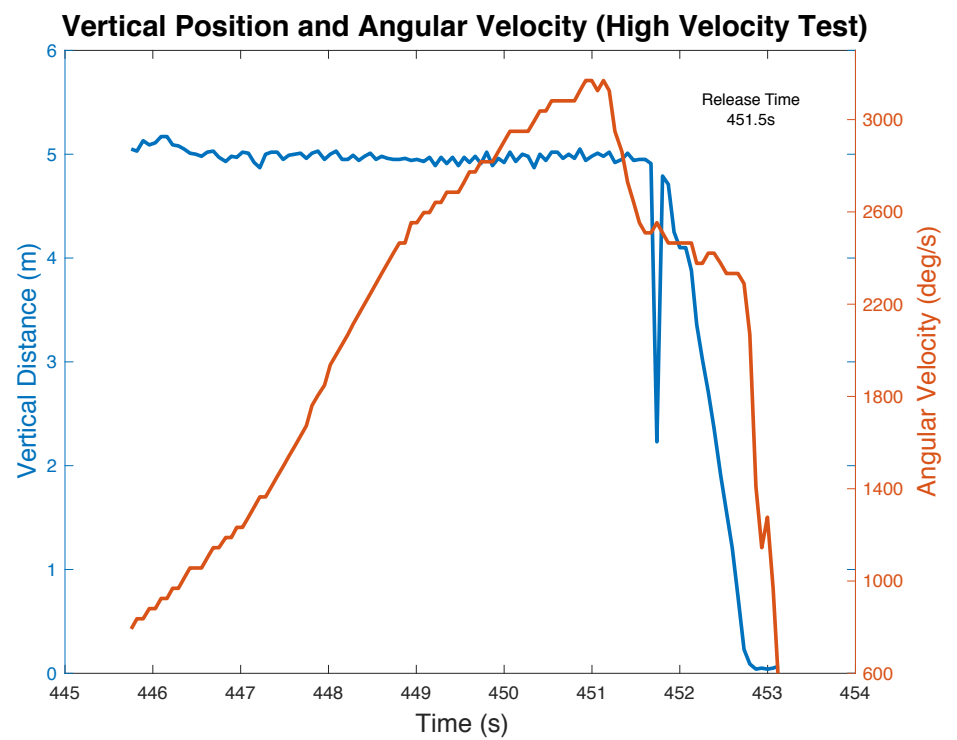

Figure 8: Raw LiDAR and angular velocity data from third drop test, released at $3200 \mathrm{deg} / \mathrm{s}$. 
the transition period between initially accelerating downwards and auto-rotation during descent.

The mass and size of the device could be reduced by altering the mass of the electronic payload, the aluminium hub and the airfoils. The DAQ system payload contributes roughly half the mass of the entire device due to the electronics, batteries and 3D printed parts. The payload mass and size could be significantly reduced by using smaller and lighter electronics. The mass of the aluminium hub could be reduced significantly by using a plastic material. After reducing the mass of the payload and hub, the length of each airfoil could be reduced and an adequate scan pattern could still be achieved.

\subsection{Point Cloud Data}

A simple, uncorrected 3D point cloud was generated by plotting collected horizontal range measurements in three dimensional space. At each time stamp $(i)$ the DAQ system sampled the horizontal range sensor for the distance to the cavity wall $\left(r_{i}\right)$, the vertical range sensor for the distance to the floor $\left(z_{i}\right)$, and the gyroscope for the angular velocity $\left(\Theta_{i}\right)$. As a first estimate, the angle rotated by the device at instant i $\left(\phi_{i}\right)$ was determined by integrating previous gyroscope measurements. The position of the cavity wall in relation to the position of the device in three dimensional space was then determined by computing

$$
x_{i}=r_{i} \cos \phi_{i} \quad y_{i}=r_{i} \sin \phi_{i} \quad z_{i}=z_{i}
$$

Plotting each sampled measurement of $i$ from 0 to the total number of samples results in a rough $3 \mathrm{D}$ point cloud of the environment surrounding the device during its flight.

One 3D point cloud was generated from each drop test to assess the devices repeatability and the effects of initial angular velocity on the helical scan pattern. The point cloud for each is illustrated by showing three section views: a top, side and front view. A 2D model of each section view is shown in Figures 9, 11 and 13. The corresponding 2D sections of each collected point cloud are shown in Figures 10, 12 and 14.

The results show that the data collected from the prototype is suitable for synthesizing a point cloud that reasonably resembles the surrounding environment. The section views of the point cloud clearly outline the boundaries of the cavity and display the helical scan pattern. All figures showing a single section view closely match one another. This shows that the device is capable of collecting repeatable results over multiple trials. The point clouds displayed show that the auto-rotating device is likely capable of providing useful information for determining a volume estimate of the cavity and determining interesting features, although further work on the fusion of collected data is warranted to provide improved results from post-processing (which is beyond the scope of this paper).

Comparing the point clouds generated from when the device was released at a high angular velocity to when it was released at a low angular velocity shows the difference in the pitch of the helical scan. When released at a high angular velocity the helical pitch 


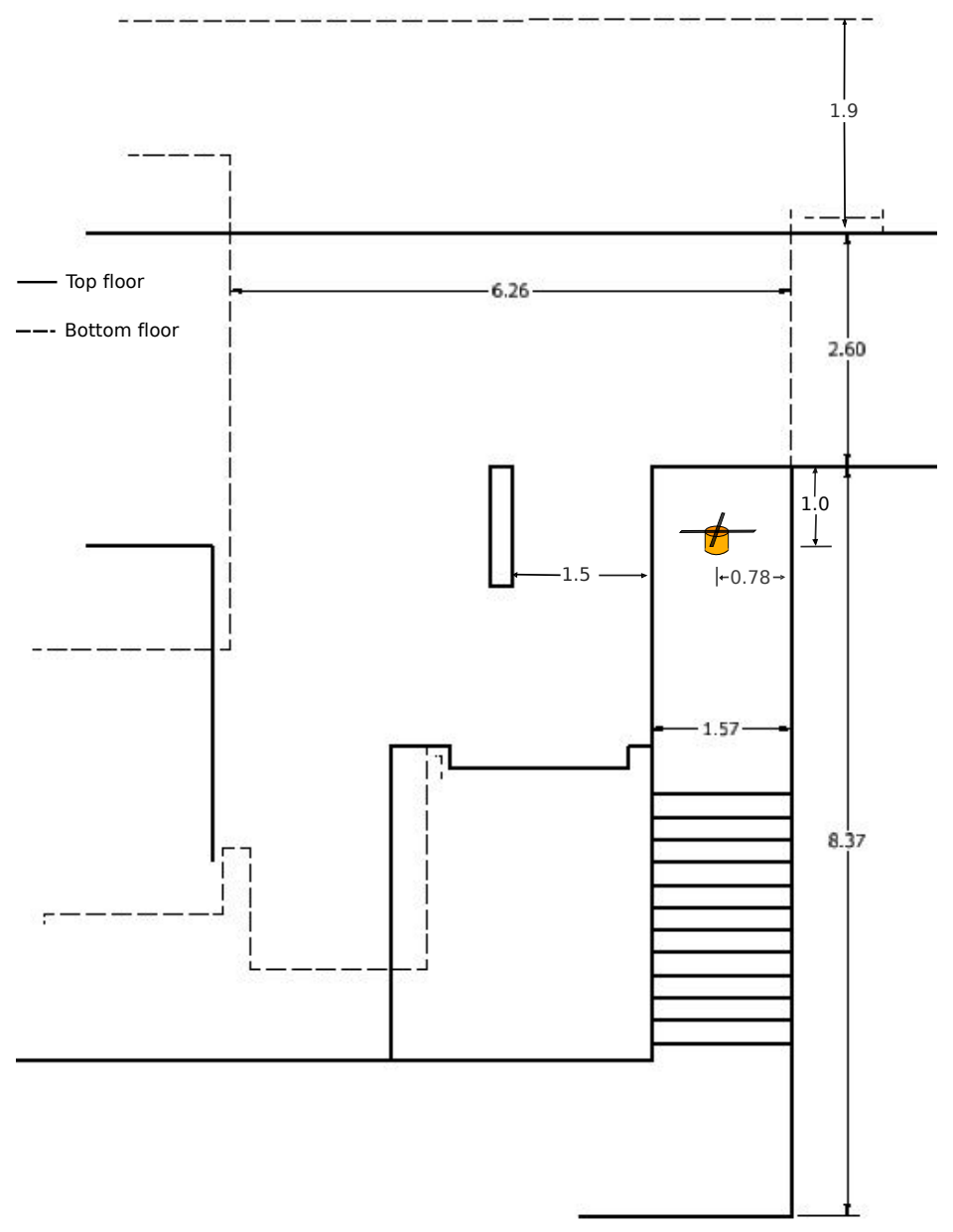

Figure 9: Top view of testing environment, displaying the bottom floor outline in dashed lines. 


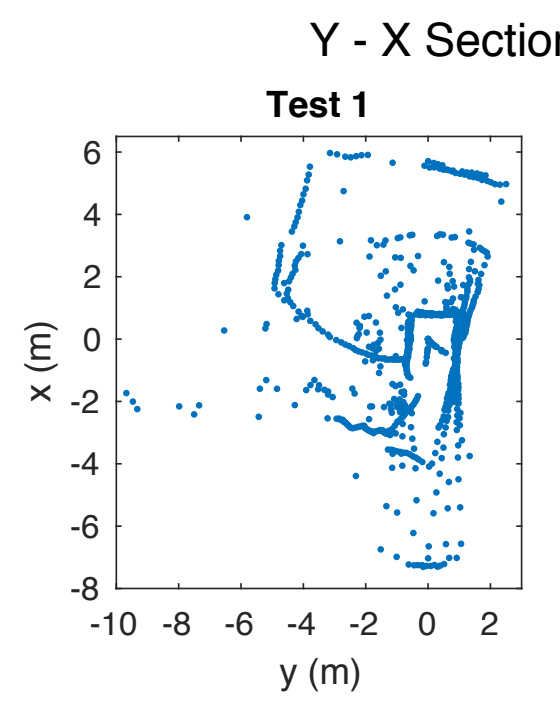

(4 Tests)
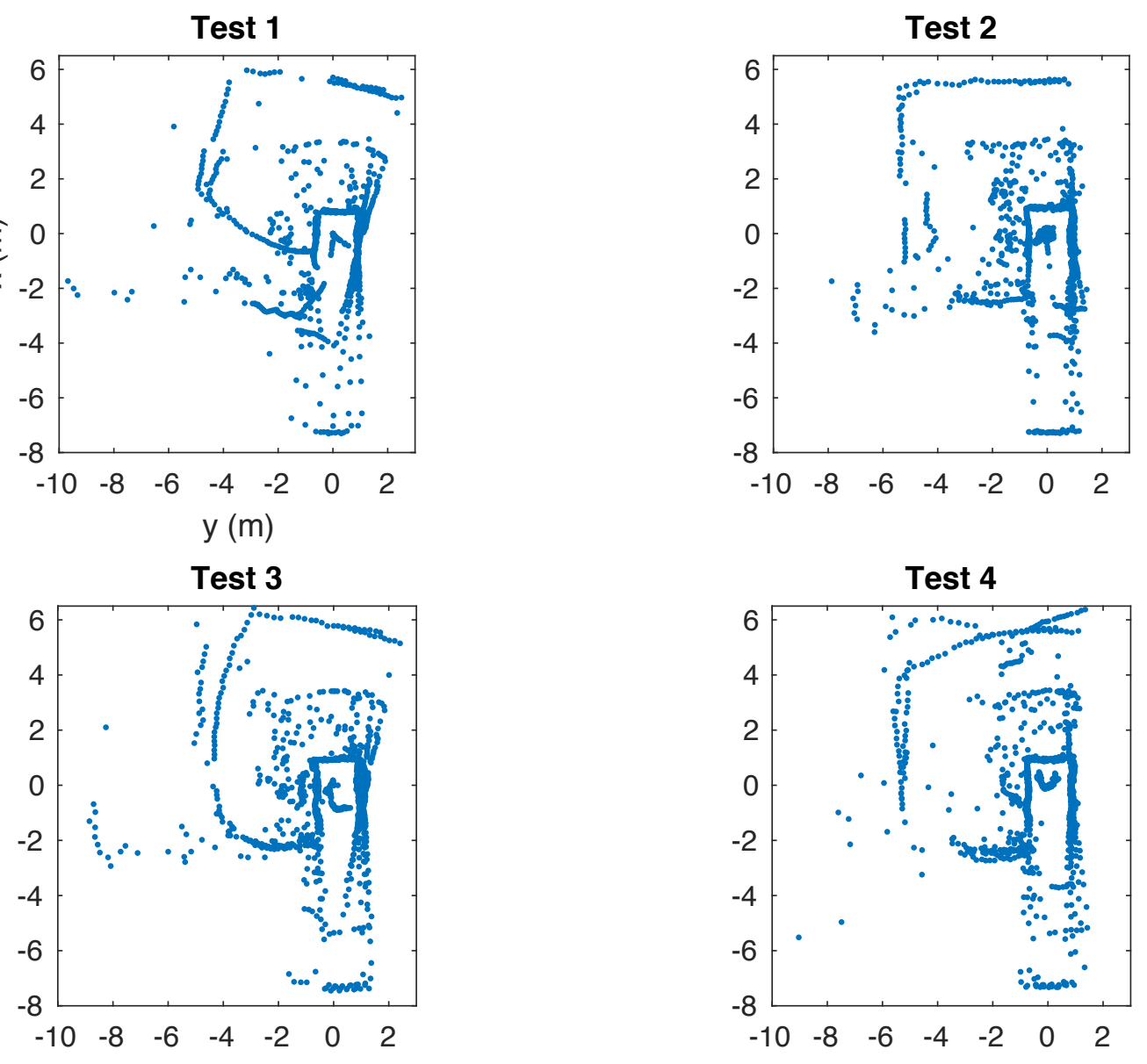

Figure 10: Top view of 3D point cloud generated from each drop test. Top left and right tests were released at low angular velocity, bottom left and right released at high angular velocity. 


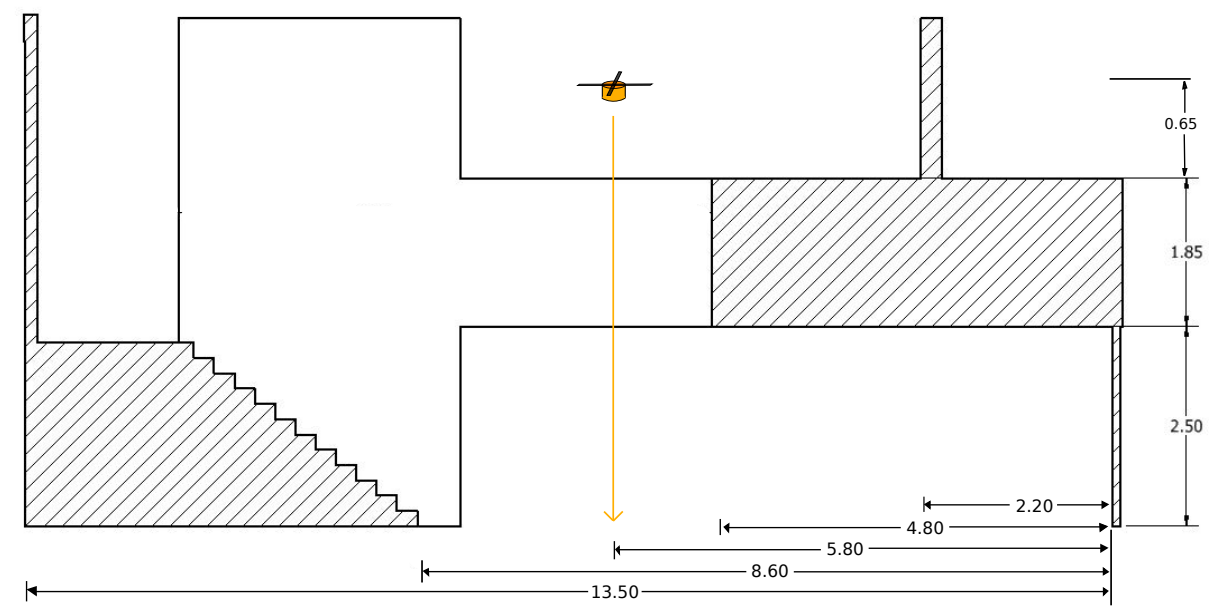

Figure 11: Side view of testing environment.

was roughly $45 \mathrm{~cm}$ and when released at a low angular velocity it was roughly $70 \mathrm{~cm}$. Increasing the angular velocity resulted in a smaller pitch and a larger point density scan. This is favourable for increasing survey accuracy as more data is collected throughout the scan.

Inaccuracies in the generated point clouds result from angular measurements being interpolated at the same frequency as the horizontal lidar sensor and drift in the gyroscope measurement. Figure 10 displays these inaccuracies as the boundaries of the cavity rotate slightly and straight walls are depicted as curves. The maximum drift in the gyroscope measurement was 13 degrees and is seen in Test 1 . Test 1,3 , and 4 show straight walls depicted as curves due to interpolated gyroscope measurements matching the frequency of the horizontal LiDAR sensor. Test 2 shows the most accurate map displaying major wall features with an accuracy of $\pm 10 \mathrm{~cm}$. Figure 10 depicts noise due to the various objects surrounding the device during its first rotation in free fall. Various objects included the railing surrounding the overhang, the hand pallet truck, and various people standing watching the experiment. Accuracy of the 3D map could be improved by calibrating the gyroscope more precisely and sampling gyroscope measurements at the same rate as the horizontal LiDAR sensor. A more precise microcontroller and gyroscope would allow these improvements to be made.

A real underground cavity presents more complications than the laboratory environment used to initially test the device. Various complications associated with deploying an auto-rotating platform in a real underground cavity include: air currents from ventilation, harsh humidity and temperature conditions, jagged rock or water at the cavity bottom, and abnormal rock outcrops. Underground cavities are also often hard to access and limit the possibility of properly placing the device before being released. Future tests are to be 


\section{X - Z Section of Helical Map (4 Tests)}
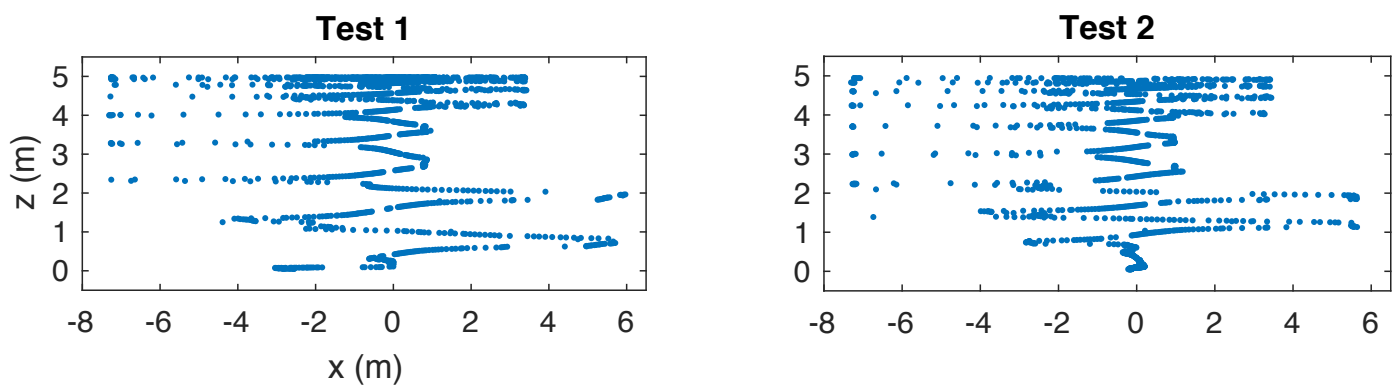

Test 3

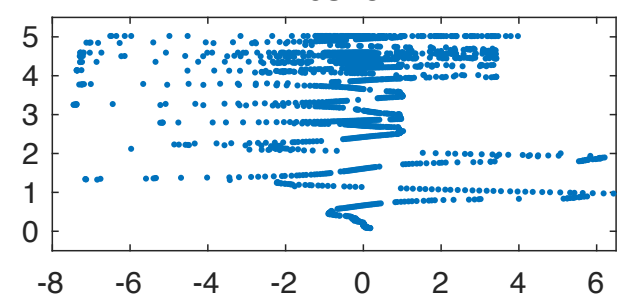

Test 4

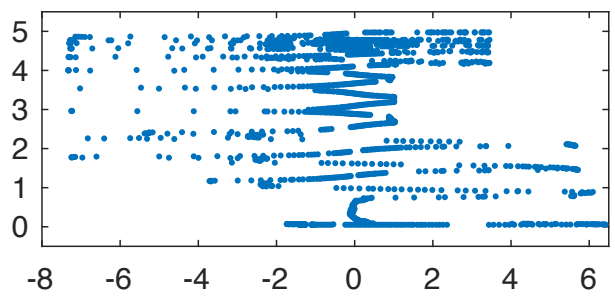

Figure 12: Side view of 3D point cloud generated from each drop test. Top left and right tests were released at low angular velocity, bottom left and right released at high angular velocity. 


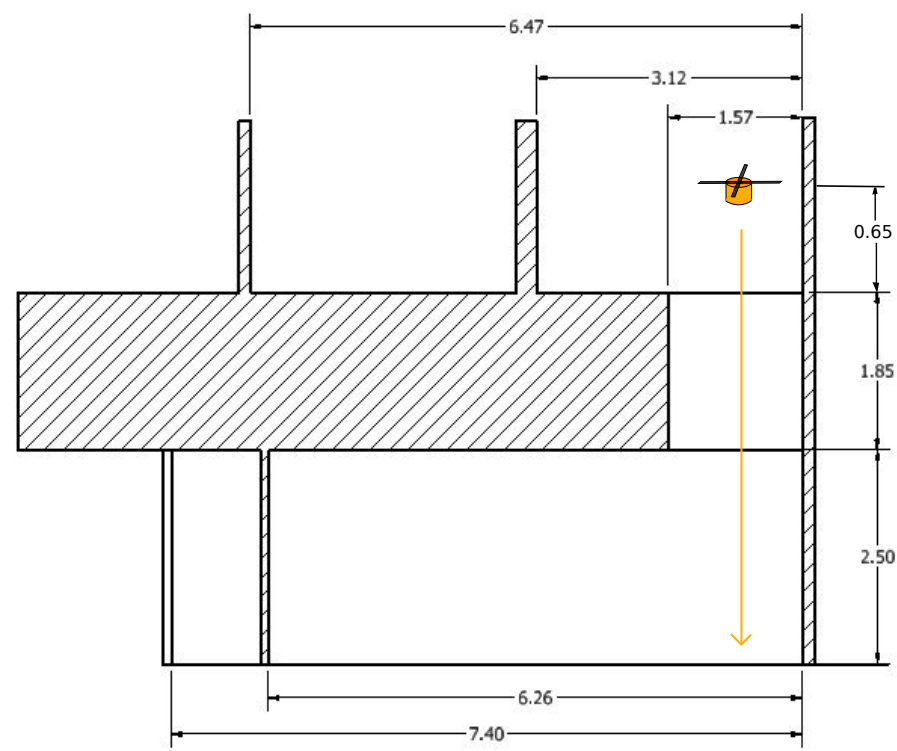

Figure 13: Front view of testing environment.

conducted in a real underground cavity.

\section{Conclusions}

1) An auto-rotating platform, equipped with a LiDAR instrumented data acquisition system that rotates as it falls is capable of collecting a 3D helical point cloud of an indoor cavity. The collected point cloud could be used to determine a volume estimate of the cavity and identify interesting features.

2) The prototype did not exhibit equilibrium auto-rotative descent during any of its four drop tests. The device accelerated downwards and decreased its angular velocity during each test.

3) The prototype design was able to maintain its integrity for multiple drop tests. No parts were damaged upon landing impact and the on-board electronic devices were not harmed or corrupted. Future focus should be on making the device smaller and lighter.

4) Based on the generated preliminary point clouds, an auto-rotating platform presents a novel opportunity for underground cavity surveying that could potentially offer a cheaper and more accurate solution for gathering point cloud data of underground cavities. 


\section{Y - Z Section of Helical Map (4 Tests)}
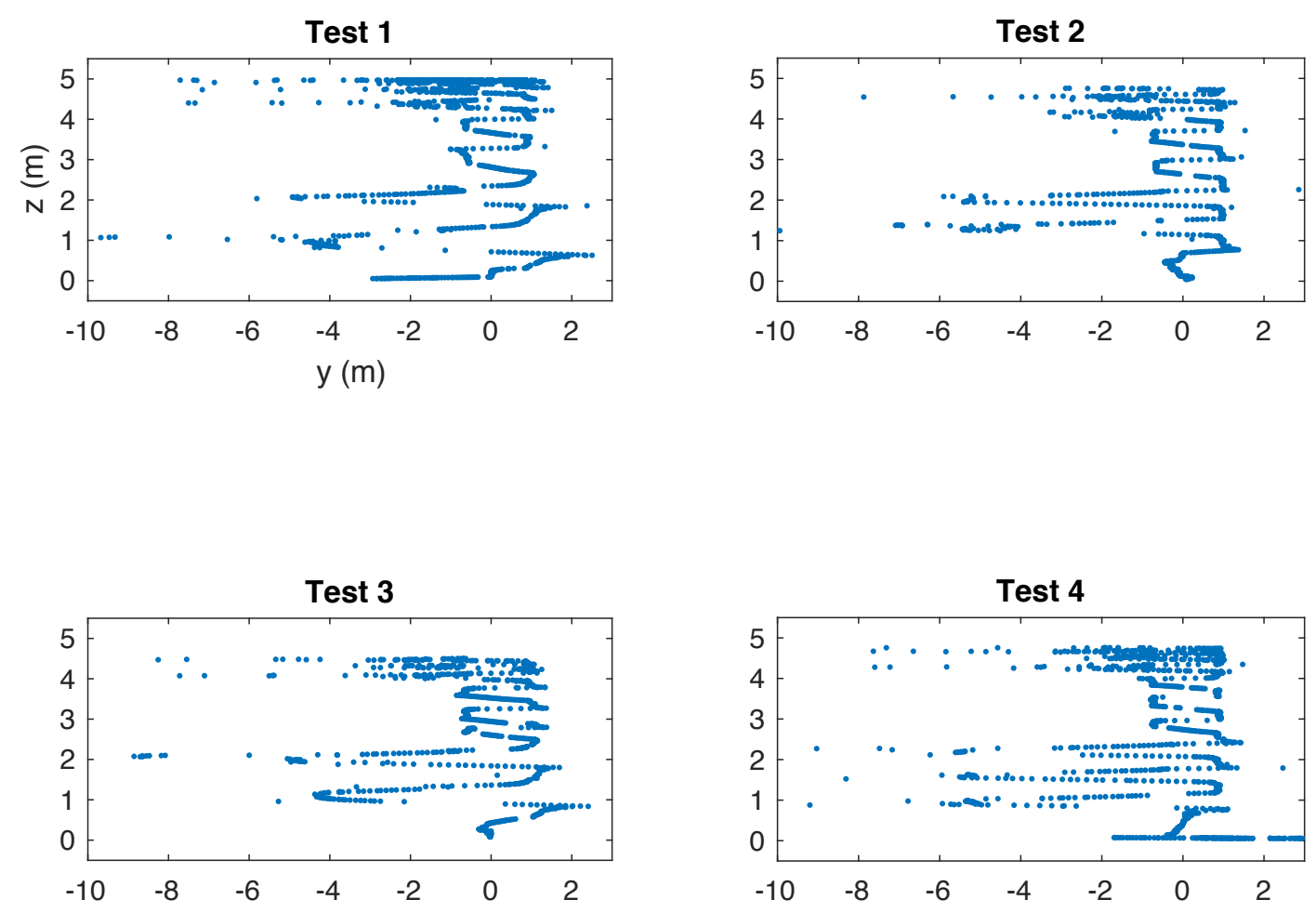

Figure 14: Front view of 3D point cloud generated from each drop test. Top left and right tests were released at low angular velocity, bottom left and right released at high angular velocity. 


\subsection{Future Research}

Future work includes improving the physical design of the prototype, further developing the post processing algorithm, and conducting tests in a real underground cavity.

A second prototype will be constructed to better resemble the initial concept of operations outlined in the paper and ensure that auto-rotation is achieved during descent. This can be achieved by significantly reducing the size and mass of the device because less airflow is required to provide enough torque to spin the rotor. The payload DAQ system and housing could be reduced in size by using a custom printed circuit board (PCB) as a microcontroller instead of the Arduino Uno. The PCB could be made into a circular shape instead of the rectangular Arduino that restricts the housing from being smaller. With a smaller payload, the rotor system could be reduced in size and aluminium parts could be replaced with lighter plastic parts.

Aside from improving the hardware, the point cloud accuracy could be improved by implementing more sophisticated post processing routines. Data processing could be improved by using sensor fusion techniques and perhaps SLAM-based mapping algorithm (e.g., such as [3] or similar) to create a more representative point cloud. Implementation of these algorithms could be done by comparing successive scan rotations or by dropping multiple devices in the cavity and comparing multiple data sets.

After a second prototype has been constructed and the processing algorithm updated, it should be tested in a real underground cavity with a stationary CMS and a mobile mapping system such as a UAV. The results from each surveying device should be compared to highlight the advantages and disadvantages of each method. This experiment would quantify the accuracy of the auto-rotating platform compared to conventional surveying methods and identify design flaws that could be adapted in future iterations.

\section{Acknowledgement}

This research was supported in part by the Natural Sciences and Engineering Research Council of Canada (NSERC) under grant RGPIN 2015 04025. Thanks also to Örebro University, Sweden and the Centre for Applied Autonomous Sensor Systems (AASS) for helping to support this research.

\section{References}

[1] J. Artieda, J. Sebastian, P. Campoy, J. Correa, I. Mondragón, C. Martínez, and M. Olivares. Visual 3-d slam from uavs. Journal of Intelligent and Robotic Systems, 55(4-5):299, 2009. 
[2] F Azhari, S. Kiely, C. Sennersten, C. Lindley, M. Matuszak, and S. Hogwood. A comparison of sensors for underground void mapping by unmanned aerial vehicles. In M Hudyma and Y Potvin, editors, Proceedings of the First International Conference on Underground Mining Technology, volume 4, pages 419-430. Australian Centre for Geomechanics, 2017.

[3] Michael Bosse, Robert Zlot, and Paul Flick. Zebedee: Design of a spring-mounted 3-d range sensor with application to mobile mapping. IEEE Transactions on Robotics, 28(5):1104-1119, oct 2012.

[4] A. Brindejonc. Design and testing of an autorotative payload delivery system: The autobody. Master's thesis, University of Maryland, 2005.

[5] Nader Fardin, Q Feng, and Ove Stephansson. Application of a new in situ 3d laser scanner to study the scale effect on the rock joint surface roughness. International Journal of Rock Mechanics and Mining Sciences, 41(2):329-335, 2004.

[6] S. Grzonka, G. Grisetti, and W. Burgard. A fully autonomous indoor quadrotor. IEEE Transactions on Robotics, 28(1):90-100, 2012.

[7] F Hadjigeorgiou et al. Ore pass practice in canadian mines. Journal of the Southern African Institute of Mining and Metallurgy, 105(11):809-816, 2005.

[8] J. Henning and H. Mitri. Numerical modelling of ore dilution in blasthole stoping. International Journal of Rock Mechanics and Mining Sciences, 44(5):692-703, 2007.

[9] W. Johnson. Helicopter theory. Princeton University Press, 1980.

[10] T. Law, J. May, A. Spathis, A. Du Plessis, and A. Palmer. Blast damage and blast dilution control: the application of bulk emulsion systems at the wme st ives junction mine. Fragblast, 5(1-2):1-20, 2001.

[11] X. Liu, X. Li, F. Li, G. Zhao, and Y. Qin. 3d cavity detection technique and its application based on cavity auto scanning laser system. Journal of Central South University of Technology, 15(2):285-288, 2008.

[12] X. Liu, Z. Luo, Q. Chen, X. Zhang, and Q. Zhu. 3d dynamic monitoring of collapse area based on cms-surpac. In Computer Science and Information Technology, 2009. ICCSIT 2009. 2nd IEEE International Conference on, pages 637-641. IEEE, 2009.

[13] X. Liu, Z. Luo, B. Yang, G. Lu, S. Cao, and X. Jiang. Visible calculation of mining index based on stope $3 \mathrm{~d}$ surveying and block modeling. International Journal of Mining Science and Technology, 22(2):139-144, 2012. 
[14] Z. Luo, X. Liu, B. Zhang, H. Lu, and C. Li. Cavity 3d modeling and correlative techniques based on cavity monitoring. Journal of central south university of technology, 15(5):639-644, 2008.

[15] C. Martin, S. Yazici, S. Espley, and G. Tan. Using numerical models to quantify stope dilution. In Proceedings of the 102nd Canadian Institute of Mining and Metallurgy Annual General Meeting, Toronto., pages 1-8, 2000.

[16] F. Miller and D. Jacob. Cavity monitoring system, 08 1993. US Patent US, 5235398.

[17] F. Miller, Y. Potvin, and D. Jacob. Laser measurement of open stope dilution. CIM Bulletin, 85(962):96-102, 1992.

[18] J. Mitchell. The mapkey: Preliminary design, construction and testing of a novel uav platform for cavity surveying. Master's thesis, Queen's University, 2017.

[19] J. Mitchell and J. Marshall. Design of a novel auto-rotating uav platform for underground mine cavity surveying. In Proceedings of the 2017 SME Annual Conference \& Expo and CMA's 119th National Western Mining Conference \& Exhibition. SME, 2017.

[20] D. Mubita. Recent initiatives in reducting dilution at konkola mine, zambia. Journal of the Southern African Institute of Mining and Metallurgy, 105(2):107-112, 2005.

[21] E. Nocerino, F. Menna, F. Remondino, I. Toschi, and P. Rodríguez-Gonzálvez. Investigation of indoor and outdoor performance of two portable mobile mapping systems. In Videometrics, Range Imaging, and Applications XIV, volume 10332, page 103320I. International Society for Optics and Photonics, 2017.

[22] C. Sennersten, C. Lindley, and A. Davie. Unmanned aerial robots for remotely operated and autonomous surveying in inaccessible underground mine voids. In Proceedings of the Third International Future Mining Conference. AusIMM, 2015.

[23] D. Seter and A. Rosen. Stability of the vertical autorotation of a single-winged samara. Journal of applied mechanics, 59(4):1000-1008, 1992.

[24] G. Turner. Towards mapping of rock walls using a uav-mounted $2 \mathrm{~d}$ laser scanner in gps denied environments. Master's thesis, Queen's University, 2016.

[25] R. Vanderbeck. A bayesian approach to convergence detection in underground excavations using lidar. Master's thesis, Queen's University, 2016.

[26] C. Watson. Towards estimating friction factors of mine drifts from low density point clouds. Master's thesis, Queen's University, 2016. 